\title{
Implantação das Diretrizes Curriculares Nacionais nos cursos de Odontologia: opinião de formandos de uma universidade pública
}

\author{
Fernanda Tenório Lopes Barbosa*; Sarah Rozette Teixeira**; Maria de Fátima Nunes***; Maria do \\ Carmo Matias Freire****
}

\author{
* Mestranda da Faculdade de Odontologia da Universidade Federal de Goiás \\ ** Cirurgiã-Dentista graduada pela Faculdade de Odontologia da \\ Universidade Federal de Goiás \\ *** Docente da Faculdade de Odontologia da Universidade Federal de Goiás
}

Recebido em 11/08/2016. Aprovado em 18/11/2016.

\begin{abstract}
RESUMO
Este estudo investigou a opinião de formandos em Odontologia de uma universidade pública sobre as Diretrizes Curriculares Nacionais (DCN) implantadas no curso. Foi realizado um estudo transversal quanti-qualitativo com aplicação de questionário aos primeiros concluintes do curso (em 2010, 2011 e 2012) após a implantação do novo currículo em uma universidade pública da região Centro-Oeste em 2006. Foi realizada estatística descritiva e análise de conteúdo temática. Dos 158 respondentes (taxa de resposta $=98,8 \%$ ), 60,8\% relataram que já tinham ouvido falar das DCN e $36,7 \%$ tinham lido total ou parcialmente o documento das diretrizes. 63,9\% dos formandos tiveram uma opinião negativa sobre a mudança curricular e esta foi associada a não leitura das DCN. Na análise qualitativa das justificativas para as opiniões, três categorias emergiram: estrutura curricular, processos de ensino-aprendizagem e perfil do egresso. As opiniões foram conflitantes na maioria dos aspectos e revelaram questões que necessitam de maior aprofundamento. Parte dos problemas ou equívocos apontados pelos estudantes referem-se à operacionalização do curso e requerem medidas de ajustes para sua superação. Concluiu-se que houve pouco conhecimento das DCN e predomínio de opiniões negativas acerca do currículo vigente entre os formandos.

Descritores: Currículo. Odontologia. Educação em Odontologia.
\end{abstract}

\section{INTRODUÇÃO}

Mudanças curriculares em odontologia têm ocorrido em diversos contextos socioeconômicos e culturais nas últimas décadas ${ }^{1-3}$. Com base na Lei de Diretrizes e Bases da Educação Nacional $(\mathrm{LDB})^{4}$, Diretrizes Curriculares Nacionais (DCN) para os Cursos de Graduação em Odontologia foram estabelecidas em 2002 pelo Ministério da Educação ${ }^{5}$. Este marco deu início à operacionalização da mudança curricular nas diversas instituições do país. Segundo as DCN, o principal objetivo dos cursos deve ser formar cirurgiões-dentistas com conhecimentos, habilidades e competências, a partir de princípios, fundamentos, condições e procedimentos que atendam às necessidades sociais 5 .

O processo de implementação das DCN em todo o país foi apoiado pela Associação 
Brasileira de Ensino Odontológico (ABENO) ${ }^{6}$. Buscando colaborar na formação de recursos humanos para o SUS e efetivar as mudanças propostas, o Ministério da Saúde, em conjunto com o Ministério da Educação, instituiu o Programa Nacional de Reorientação da Formação Profissional em Saúde (Pró-Saúde). O programa envolveu apoio financeiro aos cursos de odontologia que tiveram projetos inovadores aprovados por uma comissão assessora ${ }^{7}$.

Estas iniciativas e os resultados da implementação dos novos currículos devem ser constantemente avaliados pelas instituições de ensino. Contudo, são poucos os trabalhos publicados que abordam aspectos avaliativos e todos foram realizados em instituições das regiões Sul e Sudeste do país ${ }^{8-12}$.

Docentes, estudantes, técnicos, gestores de serviços e usuários estão envolvidos nos processos de mudança curricular e recebem influência de seus resultados. Dessa forma, possuem potencial de opinião e avaliação do currículo em implantação. Alguns estudos têm sido realizados na perspectiva dos estudantes, que são o principal objetivo das mudanças quanto à formação de recursos humanos ${ }^{8,9,12-15}$. Dentre os aspectos analisados, destacam-se o estado atual do curso, o desempenho em disciplinas específicas, e comparações entre currículo novo e antigo. Contudo, nenhum questionou a opinião dos estudantes de uma forma geral e objetiva ao final do curso, no sentido de identificar suas percepções acerca das mudanças.

$\mathrm{Na}$ Faculdade de Odontologia da Universidade Federal de Goiás (FO/UFG), criada em 1945, o atual currículo foi implantado em $2006^{16}$. No mesmo período, a referida instituição de ensino foi contemplada com o Pró-Saúde ${ }^{7}$ e mais tarde por outra importante estratégia interministerial do Governo Federal, o Programa de Educação pelo Trabalho para a Saúde (PETSaúde $)^{17}$. A proposta curricular, que foi resultado de uma ampla discussão envolvendo todos os segmentos da instituição, teve como princípios gerais: integralidade da atenção, interdisciplinaridade, integração de conteúdos, aproximação da teoria e prática e dos ciclos básico e clínico. Em síntese, o novo currículo manteve-se organizado por disciplinas, com reorganização do fluxo curricular, redistribuição de carga horária e abordagem de novos conteúdos direcionados às habilidades e competências exigidas pelas $\mathrm{DCN}^{5,16}$.

Considerando a importância da implantação das DCN e do monitoramento da sua repercussão em todo o país, este estudo investigou a opinião de formandos em odontologia de uma universidade pública sobre as DCN implantadas no curso no início dos anos 2000.

\section{METODOLOGIA}

Estudo observacional transversal, utilizando abordagens quantitativa e qualitativa. Participaram estudantes da FO/UFG ao final da graduação, nos anos de 2010, 2011 e 2012, as quais foram as três primeiras turmas que vivenciaram o atual currículo implantado naquela universidade em 2006. Foram excluídos da amostra os estudantes que tinham iniciado o curso em outras instituições e aqueles de nacionalidade estrangeira.

O projeto foi aprovado pelo Comitê de Ética em Pesquisa da UFG, parecer $n^{\circ}$ 085/06 e os participantes assinaram um Termo de Consentimento Livre e Esclarecido, concordando em participar da pesquisa de forma anônima.

Os dados foram coletados por meio de questionário autoaplicável, elaborado para uma pesquisa mais ampla a respeito dos comportamentos em saúde, motivações e percepções dos estudantes de odontologia da referida instituição. Os questionários foram 
aplicados em sala de aula no último mês letivo de cada ano. Para evitar viés de mensuração, não houve presença de docentes no momento da aplicação do instrumento, o qual foi aplicado por duas estudantes de iniciação científica. Os respondentes tiveram acesso a um documento impresso contendo o perfil do egresso e as competências e habilidades estabelecidas nas DCN, mas nenhum outro material sobre o antigo currículo foi disponibilizado.

As variáveis analisadas foram demográficas (idade e sexo), ano de conclusão do curso, conhecimento e leitura prévia do documento das $\mathrm{DCN}^{2}$ e opinião acerca da mudança curricular. A opinião foi obtida em duas questões. A questão fechada ("Qual é a sua opinião sobre a mudança curricular implementada em 2006?"), tinha as seguintes opções de resposta: "Foi positiva / Foi negativa / Ficou a mesma coisa". Como a nova matriz já estava implantada desde o início do curso, as opiniões dos formandos sobre a mudança foram aqui interpretadas como opiniões acerca do próprio currículo, refletindo também a ideia da mudança, já que estavam em vigor o antigo e o novo currículo.

Os dados quantitativos foram submetidos à análise descritiva no programa Statistical Package for the Social Sciences (SPSS 16.0). Para testar associações entre as opiniões sobre a mudança curricular (negativa e positiva) e as características dos estudantes, foi utilizado o Qui-quadrado de Pearson, com nível de significância de $5 \%$.

$\mathrm{Na}$ questão aberta, os estudantes eram solicitados a justificar suas respostas e fazer os comentários que desejassem. A análise das respostas foi realizada pelo método da Análise de Conteúdo Temática, proposta por $\operatorname{Bardin}^{18} \mathrm{e}$ revisitada por Minayo ${ }^{19}$. Esta é uma variação da análise de conteúdo e tem como conceito central o tema, que é a 'unidade de significação' extraída das falas dos participantes da pesquisa e, em seguida, confrontada com a teoria na qual é embasada (Bardin, 2011) ${ }^{18}$.

A etapa inicial consistiu em leitura flutuante das respostas dos estudantes, individualmente, por duas pesquisadoras. As respostas foram então lidas e discutidas exaustivamente, visando obter um conhecimento aprofundado da percepção dos estudantes a respeito do currículo no qual foram formados, tendo como parâmetro as indagações iniciais. Em seguida, foi realizada a categorização, que é a identificação de expressões ou palavras significativas, em função das quais o conteúdo de uma fala é organizado.

\section{RESULTADOS E DISCUSSÃO}

Dos 160 estudantes elegíveis para a pesquisa, 158 responderam o questionário (taxa de resposta $=98,8 \%$ ). A idade variou de 21 a 31 anos $(23,3 \pm 1,7)$ e $65,8 \%$ era do sexo feminino. Um total de 96 estudantes $(60,8 \%)$ relataram que já tinham ouvido falar das DCN. Quanto à leitura do documento das diretrizes, 49 (31\%) tinham lido parcialmente e nove $(5,7 \%)$ totalmente.

A grande maioria dos respondentes $(94,2 \%)$ apresentou alguma opinião sobre a mudança curricular e grande parte $(63,9 \%)$ considerou que esta foi negativa. Duas categorias foram acrescentadas pelos próprios respondentes: "Teve pontos positivos e negativos" e "Sem opinião formada". Os resultados das frequências por turma estão na tabela 1. Os percentuais de respostas oscilaram, não apresentando tendências de aumento ou redução ao longo dos anos.

Mudanças em qualquer setor geram impactos, positivos ou negativos, como podem também gerar resistências. Apesar das mudanças ocorridas na FO/UFG não terem sido radicais, já que $o$ modelo de ensino-aprendizagem tradicional foi mantido, o sentimento, por parte 
dos estudantes, de serem "cobaias" do novo currículo, pode ter contribuído para deixá-los inseguros quanto à formação que receberam. Os estudantes pesquisados não vivenciaram uma mudança propriamente nos seus currículos, pois a nova matriz já estava implantada desde o início do curso e foi mantida. Assim, as opiniões sobre a mudança podem ser interpretadas como opiniões sobre a ideia da mudança, que esteve presente de uma forma ou de outra durante a sua formação.

A convivência com colegas do antigo currículo na mesma instituição e a influência dos docentes que tiveram resistência à mudança também podem ter contribuído para suas opiniões.

Tabela 1. Opiniões dos formandos sobre a mudança curricular

\begin{tabular}{lcccc}
\hline Opiniões & $\begin{array}{c}\text { Turma 1- } \\
\text { Ano 2010 } \\
\mathbf{n}(\boldsymbol{\%})\end{array}$ & $\begin{array}{c}\text { Turma 2- } \\
\text { Ano 2011 } \\
\mathbf{n}(\boldsymbol{\%})\end{array}$ & $\begin{array}{c}\text { Turma 3- } \\
\text { Ano 2012 } \\
\mathbf{n}(\boldsymbol{\%})\end{array}$ & $\begin{array}{c}\text { Total } \\
\mathbf{n}(\boldsymbol{\%})\end{array}$ \\
\hline Foi positiva & $12(26,8)$ & $8(15,1)$ & $12(23,1)$ & $32(20,1)$ \\
Foi negativa & $32(56,1)$ & $38(71,7)$ & $31(59,6)$ & $101(63,9)$ \\
Ficou a mesma coisa & $2(4,9)$ & $2(3,8)$ & $4(7,6)$ & $8(5,1)$ \\
Teve pontos positivos e negativos & $4(7,3)$ & $1(1,9)$ & $3(5,8)$ & $8(5,1)$ \\
Sem opinião formada & $3(4,9)$ & $2(3,8)$ & $2(3,9)$ & $7(4,5)$ \\
Não responderam & 0 & $2(3,8)$ & 0 & $2(1,3)$ \\
\hline Total & $\mathbf{5 3 ( 1 0 0 )}$ & $\mathbf{5 3 ( 1 0 0 )}$ & $\mathbf{5 2 ( 1 0 0 )}$ & $\mathbf{1 5 8}(\mathbf{1 0 0})$ \\
\hline
\end{tabular}

Comparações com estudos anteriores devem ser feitas com ressalvas, pois os contextos e métodos utilizados são diversos e nenhum fez o mesmo questionamento do presente estudo. De modo geral, nossos resultados contrastam com aqueles verificados em outras instituições, nas quais as percepções dos estudantes sobre a sua formação foram mais positivas. No estudo de Junges et al. ${ }^{8}$, por exemplo, houve comparação entre o novo e o antigo, e uma melhor auto avaliação foi verificada entre os formandos do novo currículo. Em outro estudo ${ }^{15}$, foram incluídos estudantes de todos os períodos do curso. A ausência de docentes no momento da aplicação do questionário do presente estudo também pode ter influenciado as respostas, deixando os estudantes mais à vontade para expressarem suas opiniões, em comparação com os demais estudos nos quais o docente esteve presente.

Os resultados da análise da associação entre as categorias de opinião e as demais variáveis investigadas estão na tabela 2 . Apenas a leitura prévia das DCN apresentou associação significativa com a opinião dos formandos sobre a mudança curricular. O percentual de formandos que tiveram avaliação positiva foi mais elevado entre aqueles que relataram ter lido total ou parcialmente o documento das DCN (antes da pesquisa), em comparação com aqueles que relataram não ter lido $(\mathrm{p}<0,05)$. Este achado tem importantes implicações para os futuros processos de mudanças curriculares, pois mostram que o conhecimento das diretrizes pelos estudantes pode melhorar ou ampliar a sua compreensão acerca destes processos e influenciar positivamente na sua percepção sobre o currículo como um todo. 
Tabela 2. Opiniões dos formandos segundo variáveis demográficas, conhecimento e leitura das Diretrizes Curriculares Nacionais

\begin{tabular}{|c|c|c|c|c|}
\hline \multirow[b]{2}{*}{ Variáveis } & \multicolumn{3}{|c|}{ Opiniões dos estudantes $(\mathrm{n}=133) *$} & \multirow[b]{2}{*}{$\begin{array}{l}\text { Valor } \\
\text { de } \mathrm{p}^{* *}\end{array}$} \\
\hline & $\begin{array}{l}\text { Positiva } \\
\text { n (\%) }\end{array}$ & $\begin{array}{l}\text { Negativa } \\
\text { n (\%) }\end{array}$ & $\begin{array}{l}\text { Total } \\
\text { n (\%) }\end{array}$ & \\
\hline \multicolumn{5}{|l|}{ Sexo } \\
\hline Feminino & $25(28,4)$ & $63(71,6)$ & $88(100,0)$ & 0,101 \\
\hline Masculino & $7(15,6)$ & $38(84,4)$ & $45(100,0)$ & \\
\hline \multicolumn{5}{|l|}{ Idade } \\
\hline 21 a 22 & $9(22,5)$ & $31(77,5)$ & $40(100,0)$ & 0,428 \\
\hline 23 & $10(19,6)$ & $41(80,4)$ & $51(100,0)$ & \\
\hline 24 a 31 & $13(31,0)$ & $29(69,0)$ & $42(100,0)$ & \\
\hline \multicolumn{5}{|c|}{ Ano de conclusão do curso } \\
\hline 2010 & $12(27,3)$ & $32(72,7)$ & $44(100,0)$ & 0,424 \\
\hline 2011 & $8(17,4)$ & $38(82,6)$ & $46(100,0)$ & \\
\hline 2012 & $12(27,9)$ & $31(72,1)$ & $43(100,0)$ & \\
\hline \multicolumn{5}{|c|}{ Ouviu falar nas DCN } \\
\hline Sim & $18(22,8)$ & $61(77,2)$ & $79(100,0)$ & 0,677 \\
\hline Não & $14(25,9)$ & $40(74,1)$ & $54(100,0)$ & \\
\hline \multicolumn{5}{|c|}{ Leu parcial ou totalmente as DCN } \\
\hline $\operatorname{Sim}$ & $16(34,8)$ & $30(65,2)$ & $46(100,0)$ & 0,035 \\
\hline Não & $16(18,4)$ & $71(81,6)$ & $87(100,0)$ & \\
\hline
\end{tabular}

A análise de conteúdo temática foi realizada para melhor compreender os significados das justificativas apresentadas pelos formandos para suas opiniões e chegou-se a três categorias: estrutura curricular, processos de ensino-aprendizagem e perfil do egresso, com suas respectivas ideias centrais e núcleos do sentido (tabela 3 ).

As categorias foram nomeadas de acordo com o conteúdo do atual Projeto Pedagógico do Curso da FO/UFG. Nos diversos núcleos de sentido observados, aspectos positivos e negativos foram expressos pelos estudantes, os quais serão descritos a seguir.

\section{Estrutura curricular}

Na opinião dos formandos, a integralização do cuidado permitiu ter uma " [...] melhor visão e mais ampla [...]", possibilitando uma formação "[...] humanista, voltada para os serviços de saúde e incluindo promoção, prevenção e recuperação da saúde, além de envolver a atuação multiprofissional [...]".

Por outro lado, as mudanças realizadas no sentido de integrar algumas disciplinas produziu algumas percepções negativas: “(...) O currículo se tornou muito generalista (...)”. Uma dificuldade relatada com frequência pelos estudantes é a de que "[...] vários alunos estão se formando sem realizar alguns procedimentos básicos [...]", que, segundo eles, se deve a "[...] falta de clínicas individualizadas de determinadas disciplinas [...]".

As opiniões referentes à integralidade da atenção revelaram que os aspectos considerados favoráveis para o paciente não eram 
considerados favoráveis para os próprios estudantes, visto que "[...] o novo currículo deixou muito a desejar em várias áreas mais clínicas, onde se preconizou o atendimento integral do paciente e deixou de lado as especialidades, uma vez que para fazermos procedimentos de todas as áreas tivemos que pegar vários pacientes e, na maioria das vezes não terminávamos nenhum, fazendo apenas o que nos interessava [...]".

Dificuldades em oferecer atenção integral aos pacientes foram manifestadas também por formandos de outro estudo ${ }^{11}$. Contudo, de acordo as DCN, o foco principal do processo ensinoaprendizagem passa a ser as necessidades dos pacientes e não a produtividade individual dos estudantes, apontando para uma avaliação mais qualitativa dos mesmos ${ }^{10}$.

Deficiências relativas à integração curricular foram percebidas também em estudo anterior $^{12}$. Sabe-se que a interdisciplinaridade vem contribuir para superar a lógica da fragmentação dos conteúdos e práticas e fortalecer a formação do generalista ${ }^{20}$ e é fortemente recomendada ${ }^{21}$.

A importância dos projetos de extensão foi reconhecida pelos pesquisados, corroborando estudo anterior realizado em outra instituição ${ }^{11}$. Contudo, os formandos relataram que tiveram que buscar conhecimento em projetos de extensão de modo a compensar as lacunas que a clínica não supriu: "[...] os projetos de extensão são perfeitos, mas não deveriam ser usados para encobrir falhas inerentes a mudança curricular [...]".

Conforme consta na atual LDB, a extensão é uma das finalidades da educação superior e deve ser promovida de forma aberta à população ${ }^{4}$ Entretanto, a extensão deve adicionar e não suprir um conteúdo básico.

Os estágios curriculares, outro aspecto importante do ensino odontológico desde os anos
1970 e contemplado nas atuais DCN, foram reconhecidos como uma estratégia que favorece a formação dos estudantes, em concordância com estudos realizados em outros cursos do país ${ }^{11,12,15}$. Porém, foi também mencionado que "[...] os campos de estágio foram bem abrangentes, mas é preciso ser mais claros quanto aos objetivos das disciplinas [...]". Em concordância, Wernek et al. ${ }^{22}$ consideram a necessidade de se definir mais claramente o estágio curricular supervisionado, para que não seja confundido com outras práticas clínicas dentro das instituições.

Não obstante a avaliação positiva dos estágios no presente estudo, dentre eles os realizados no serviço público, muitos estudantes expressaram opiniões desfavoráveis à área de saúde coletiva. Para um dos respondentes, “[...] é extremamente necessário nosso conhecimento das diretrizes e princípios do SUS e tudo, mas é inviável tirar [...] as matérias para colocar o SUS no lugar [...]". Opiniões similares ou que denotam visão restrita acerca desta área foram manifestadas também por estudantes da região Sudeste en, $^{23,24}$.

Os resultados mostram a pouca compreensão por parte dos estudantes (e talvez do corpo docente e técnico) dos principais objetivos da manutenção de uma série de disciplinas de saúde coletiva, que são trabalhar conteúdos ao longo do curso e aumentar a aproximação dos estudantes com o serviço público de saúde. Estes aspectos constituem a ideia central do Pró-Saúde ${ }^{7}$, que financiou parte das ações necessárias às mudanças do currículo como um todo. Para Fagundes et al. ${ }^{25}$, 'não basta definir novos locais de ensino; é preciso exercitar a reflexão sobre a prática, para que esta possa ser uma referência para interpelação e transformação das formas tradicionais de conceber currículo'. Não obstante as dificuldades encontradas no ensino da Odontologia Coletiva, seus objetivos 
Tabela 3. Categorização das opiniões dos formandos sobre a mudança curricular

\begin{tabular}{|c|c|c|}
\hline Categorias & Núcleos do sentido & Ideias centrais \\
\hline \multirow{10}{*}{$\begin{array}{l}\text { Estrutura } \\
\text { curricular }\end{array}$} & $\begin{array}{l}\text { Distribuição dos } \\
\text { conteúdos }\end{array}$ & $\begin{array}{l}\text { Conhecimento gradativo; falta de sequência lógica; } \\
\text { necessidade de mais conteúdos de algumas disciplinas. }\end{array}$ \\
\hline & $\begin{array}{l}\text { Distribuição da carga } \\
\text { horária }\end{array}$ & $\begin{array}{l}\text { Déficit na carga horária de disciplinas; sobrecarga de } \\
\text { outras. }\end{array}$ \\
\hline & Treinamento clínico & $\begin{array}{l}\text { Maior número de aulas práticas; estudantes saem da } \\
\text { faculdade sem realizar procedimentos básicos. }\end{array}$ \\
\hline & $\begin{array}{l}\text { Integralidade da } \\
\text { atenção }\end{array}$ & $\begin{array}{l}\text { Tratamento integralizado do paciente; aprendizado mais } \\
\text { sedimentado; formação multiprofissional; dependência das } \\
\text { necessidades do paciente; pouca ou nenhuma prática em } \\
\text { áreas que não eram necessidade do paciente. }\end{array}$ \\
\hline & Interdisciplinaridade & $\begin{array}{l}\text { Integração entre disciplinas; planejamento integrado; visão } \\
\text { ampliada; não funciona na prática; déficit no aprendizado; } \\
\text { não houve preparo para interdisciplinaridade. }\end{array}$ \\
\hline & Extensão & $\begin{array}{l}\text { Projetos de extensão perfeitos; extensão como } \\
\text { compensação de conteúdos práticos; extensão para encobrir } \\
\text { falhas da mudaça. }\end{array}$ \\
\hline & Estágios & Campos abrangentes; objetivos não são claros. \\
\hline & Metodologia científica & Positivo para o aprendizado. \\
\hline & Odontologia Coletiva & $\begin{array}{l}\text { Conteúdo excessivo de odontologia coletiva; temas } \\
\text { exaustivos; conteúdo defasado; conteúdo repetitivo. }\end{array}$ \\
\hline & $\begin{array}{l}\text { Trabalho de Conclusão } \\
\text { de Curso }\end{array}$ & $\begin{array}{l}\text { Mudança nas regras favoreceu a realização de trabalho } \\
\text { científico. }\end{array}$ \\
\hline \multirow[t]{6}{*}{$\begin{array}{l}\text { Processos de } \\
\text { ensino- } \\
\text { aprendizagem }\end{array}$} & $\begin{array}{l}\text { Uso de metodologias } \\
\text { ativas }\end{array}$ & $\begin{array}{l}\text { Facilitou o aprendizado; independência do estudante; } \\
\text { aproximação com a realidade; visão ampliada; insatisfação } \\
\text { com o método; falta de objetivo. }\end{array}$ \\
\hline & $\begin{array}{l}\text { Apresentação dos } \\
\text { conteúdos }\end{array}$ & Superficialidade dos conteúdos \\
\hline & $\begin{array}{l}\text { Postura e desempenho } \\
\text { dos docentes }\end{array}$ & $\begin{array}{l}\text { Falta de capacitação docente para interdisciplinaridade e } \\
\text { metodologias ativas; aulas longas e cansativas; desânimo } \\
\text { dos docentes com a mudança curricular; falta de integração } \\
\text { entre docentes. }\end{array}$ \\
\hline & $\begin{array}{l}\text { Motivação e } \\
\text { desempenho dos } \\
\text { estudantes }\end{array}$ & $\begin{array}{l}\text { Busca pelo conhecimento de forma inadequada; estudante } \\
\text { pouco motivado. }\end{array}$ \\
\hline & $\begin{array}{l}\text { Sistema de avaliação do } \\
\text { estudante }\end{array}$ & $\begin{array}{l}\text { Redução da média para aprovação prejudicial ao processo } \\
\text { avaliativo. }\end{array}$ \\
\hline & $\begin{array}{l}\text { Funcionamento das } \\
\text { clínicas }\end{array}$ & $\begin{array}{l}\text { Fluxo de orientação do docente na clínica gera atrasos; } \\
\text { necessidade de cotas de procedimento. }\end{array}$ \\
\hline \multirow{3}{*}{ Perfil do egresso } & Formação humanística & Formação mais humanizada. \\
\hline & $\begin{array}{l}\text { Formação para atuar no } \\
\text { serviço público }\end{array}$ & $\begin{array}{l}\text { Mudança no perfil do profissional; a maioria não pretende } \\
\text { atuar no serviço público. }\end{array}$ \\
\hline & $\begin{array}{l}\text { Preparo para o mercado } \\
\text { de trabalho }\end{array}$ & $\begin{array}{l}\text { Despreparo; insegurança; incerteza de formação adequada } \\
\text { para atuação profissional. }\end{array}$ \\
\hline
\end{tabular}

parecem ser alcançados durante a vida coletiva ministrados durante a graduação como profissional. Num estudo realizado com egressos muito importantes ou importantes para sua do antigo currículo do mesmo curso, a grande atuação profissional, valorizou os estágios maioria reconheceu os conteúdos de saúde curriculares e desejava ter aproveitado mais as 
práticas extramuros ${ }^{26}$. Parece tratar-se, portanto, de um efeito tardio. Interesse crescente pela atuação no serviço público tem sido manifestado nesta $^{28}$ e em outra instituição no Sul do país ${ }^{8}$.

\section{Processos de ensino-aprendizagem}

$\mathrm{O}$ uso de metodologias ativas de aprendizagem, que é uma das proposições das $\mathrm{DCN}^{5}$, foi percebida de forma contraditória pelos formandos. Para alguns, o novo currículo, “[...] ajudou a procurar por nossa educação e nos tornar mais ambiciosos por conhecimento [...]". $\mathrm{O}$ aspecto positivo sugere o professor como facilitador e mediador do processo de ensino e aprendizagem e o estudante como participante ativo desse processo.

Outros respondentes mostraram insatisfação com o uso de metodologias ativas e despreparo para a independência da busca pelo conhecimento, pois se consideraram dependentes de professores. Sugerem também que houve uma banalização do conhecimento, ao perceberem que têm buscado conhecimento em fontes não confiáveis ou que têm deixado de dar valor às aulas, por acreditarem que tudo que precisam pode ser encontrado na internet. Alguns consideram que há "[...] Professores incapacitados de atuar na metodologia ativa e de modo interdisciplinar (...)”.

Para Toassi et al. ${ }^{13}$, este aspecto pode estar relacionado ao processo de transição e adaptação de um modelo centralizado no professor, que estimula a passividade e acentua a competitividade entre os estudantes, para um modelo de interação, diálogo e compartilhamento entre os sujeitos envolvidos.

A postura dos docentes parece influenciar as atitudes e opiniões dos estudantes, pois afirmam que o "[...] reflexo de desânimo de professores que não concordaram com essas mudanças acabam nos prejudicando [...]". É importante levar em consideração que as exigências atuais da formação odontológica ainda desafiam a Universidade e os atores envolvidos para que se repense a formação do professor, passando pela transformação do seu papel. As barreiras para esta transformação se apresentam sob as diversas formas. Num estudo realizado nos Estados Unidos ${ }^{28}$, os docentes relataram ter pouco tempo para desenvolver diferentes estratégias pedagógicas e esta foi a principal barreira.

\section{Perfil do egresso}

Os estudantes sentem que "o novo currículo nos permitiu uma formação mais humanizada [...]", característica ressaltada pelas DCN. Resultado similar foi encontrado em outro curso da região $\mathrm{Sul}^{12}$. Entretanto, a sensação de serem as "cobaias" do novo currículo os faz pensar que foram prejudicados e que não tiveram uma educação que os qualifiquem para o mercado de trabalho, tanto para o serviço público, como para o serviço privado. Alguns respondentes disseram estar "[...] mais preparados ao mercado de trabalho já que estagiamos e aperfeiçoamos mais no decorrer do curso [...]". Outros relataram que o novo currículo os preparou para serem apenas profissionais do serviço público.

O desafio continua sendo mudar de uma concepção e prática predominantemente individual, curativa e com foco no exercício liberal da profissão, para uma formação mais voltada para a promoção da saúde, mais integrada à saúde geral e mais adequada à atuação no serviço público ${ }^{7,29}$.

Destaca-se, como limitação do presente estudo, o fato de ter como população de estudo apenas os formandos do novo currículo, não sendo possível comparar suas percepções com as de formandos do antigo currículo. No âmbito da instituição pesquisada, os resultados foram encaminhados ao Núcleo Docente Estruturante e 
podem ser úteis para o aprimoramento do currículo em vigor. A temática não se esgota neste estudo, mas abre oportunidade a investigações relacionadas. A adequação ou não da mudança ocorrida na instituição de ensino em questão deve ser avaliada de forma mais abrangente, incluindo os demais sujeitos envolvidos, outros indicadores e instrumentos apropriados. Para que o novo currículo atinja seu objetivo mais amplo, ele precisa ser melhor compreendido pelos sujeitos implicados em sua implantação, além de ser constantemente avaliado e aprimorado.

\section{CONCLUSÕES}

Grande parte dos formandos consideraram que a mudança curricular foi negativa e esta foi associada à não leitura das DCN. As opiniões foram conflitantes na maioria dos aspectos e revelaram que há questões que necessitam de aprofundamento. Parte dos problemas ou equívocos apontados pelos estudantes, e que nortearam suas respostas, referem-se à operacionalização do curso. Estes requerem medidas de ajustes, sem necessariamente reverter a mudança curricular realizada.

\section{ABSTRACT \\ Implantation of the National Curriculum Guidelines in Dental Courses: opinions of final year students in a public university}

This study investigated opinions of final year dental students in a public university regarding the National Curriculum Guidelines (NCG) set out in the beginning of the 2000s. A cross sectional quantitative and qualitative study was conducted using a questionnaire applied to the first graduates under the new curriculum (in 2010, 2011 and 2012) of a public university in Midwest Brazil from 2006. Descriptive statistics and content analysis were carried out. Of the 158 respondents (response rate $=98.8 \%$ ), $60.8 \%$ reported they were aware of the NCG and $36.7 \%$ had read the reference document totally or in part. $63.9 \%$ of them had a negative opinion and this was associated to the lack of reading the reference document. In the qualitative analysis of the reasons for the answers, three categories emerged: curriculum structure, teaching-learning processes and graduate profile. Opinions were mostly conflicting and revealed points needing a deeper understanding. Part of the problems or equivocal aspects pointed out by the students refer to the operationalization of the course and require measures of adjustments. It was concluded that there was low awareness of the NCG reference document and predominantly negative opinions regarding the curriculum among the students.

Descriptors: Curriculum. Dentistry. Dental Education.

\section{REFERÊNCIAS}

1. Murtomaa H. Dental education in Europe. Eur J Dent Educ. 2009;3(1):1-2.

2. Haden NK, Hendricson WD, Kassebaum DK, Ranney RR, Weinstein G, Anderson EL, Valachovic RW. Curriculum change in dental education. J Dent Educ. 2010; 74(5):539-57.

3. Loomer PM, Masalu JR, Mimghaba E, Perry DA. New curriculum in dentistry for Tanzania: competency-based education for patient and population health (2008-2011). J Public Health Policy. 2012; 33(1):92-109.

4. Brasil. Lei de Diretrizes e Bases da Educação Nacional. Lei número 9394, 20 de dezembro de 1996. Diário Oficial da União 1996; 23 dez.

5. Ministério da Educação. Conselho Nacional de Educação. Câmara de Educação Superior. Resolução CNE/CES 3, de 19 de fevereiro de 2002. Institui Diretrizes Curriculares Nacionais do Curso de Graduação em Odontologia. Diário Oficial da União 2002; 04 mar.

6. Morita MC, Kriger L, Carvalho ACP, Haddad AE. Implantação das diretrizes curriculares nacionais em odontologia. Projeto de Cooperação Técnica (ABENO/ OPAS/ MS). Maringá: Dental Press; 2007.

7. Ministério da Saúde. Ministério da Educação. Portaria interministerial n. 2.101, de 3 de novembro de 2005. Institui o 
Programa Nacional de Reorientação da Formação Profissional em Saúde - PróSaúde - para os cursos de Graduação em Medicina, Enfermagem e Odontologia. Diário Oficial da União 2005.

8. Junges R, Stello RS, Portella FF, Rösing CK, Samuel SMW. Impact of the implantation of a new curriculum in the process of learning in a Faculty of Dentistry in Brazil. Braz Oral Res. 2011;25(6):478-84.

9. Vieira GM, Canto GL. Avaliação da reforma curricular do curso de odontologia da Universidade Federal de Santa Catarina. Rev ABENO. 2012;12(2):198-206.

10. Ferreti LH, Appel TG, Miguel LCM, Madeira L. Avaliação discente e as Diretrizes Curriculares Nacionais realidade das clínicas integradas da UNIVILLE. Rev ABENO. 2012;12(2):15562.

11. Fadel CB, Baldani MH. Percepções de formandos do curso de odontologia sobre as diretrizes curriculares nacionais. Trab Educ Saúde. 2013; 11(2):339-54.

12. Toassi RFC, Souza JM, Baumgarten A, Rosing CK. Avaliação curricular na educação superior em odontologia: discutindo as mudanças curriculares na formação em saúde no Brasil. Rev ABENO. 2012; 12(2):170-77.

13. Zilbovicius C, Araujo ME, Botazzo C, Frias AC, Junqueira SR, Junqueira CR. A paradigm shift in predoctoral dental curricula in Brazil: Evaluating the process of change. J Dent Educ. 2011;75(4):557-64.

14. Gabriel M, Tanaka EE. Formação profissional em Odontologia: percepção discente da interação curricular. Rev ABENO. 2011;11(1):19-22.

15. Silveira JLGC, Garcia VL. Curricular change within dentistry: meanings according to the subjects of the learning. Interface Comun Saúde Educ. 2015;19(52):145-58.

16. Universidade Federal de Goiás, Faculdade de Odontologia. Projeto Pedagógico do Curso de Odontologia da Universidade Federal de Goiás. Goiânia; 2009. [Acesso em 23 maio 2016]. Disponível em:
http://www.odonto.ufg.br/up/133/o/PPC_F O UFG.pdf.

17. Ministério da Saúde. Ministério da Educação. Portaria interministerial $\mathrm{n}^{\circ}$ 421, de 3 de março de 2010. Institui o Programa de Educação pelo Trabalho para a Saúde (PET Saúde) e dá outras providências. Diário Oficial da União 2010; 5 mar.

18. Bardin L. Análise de conteúdo. Lisboa: Edições 70; 2011.

19. Minayo MCS. O desafio do conhecimento. Pesquisa qualitativa em saúde. $14^{\mathrm{a}}$ Ed. São Paulo: HUCITEC; 2014.

20. Lemos CLS, Fonseca SG. Saberes e práticas curriculares: um estudo de um curso superior na área da saúde. Interface Comun Saúde Educ. 2009;3(28):57-69.

21. Haden NK, Hendricson WD, Kassebaum DK, Ranney RR, Weinstein G, Anderson EL, Valachovic RW. Curriculum change in dental education. J Dent Educ. 2010; 74(5):539-57.

22. Wernek MAF, Senna MIB, Drumond MM, Lucas SD. Nem tudo é estágio: contribuições para o debate. Ciênc Saúde Coletiva. 2010; 15(1):221-31.

23. Costa SM, Silveira MF, Durães SJA, Abreu MHNG, Bonan PRF. Perceptions of dental students regarding dentistry, the job market and the public healthcare system. Ciênc Saúde Coletiva. 2012;17(5):1285-96.

24. Moimaz, SAS. et al. Representação social de acadêmicos de odontologia sobre a área de Odontologia Social. Rev ABENO. 2006; 6(2):145-9.

25. Fagundes NC, Burnham TF. Discutindo a relação entre espaço e aprendizagem na formação de profissionais de saúde. Interface Comun Saúde Educ. 2005;9(16) :105-14.

26. Badan DEC, Marcelo VC, Rocha DG. Percepção e utilização dos conteúdos de saúde coletiva por cirurgiões-dentistas egressos da Universidade Federal de Goiás. Ciênc Saúde Coletiva. 2010; 15(supl.1) :1811-18.

27. Freire MCM, Jordão LMR, Ferreira NP, Leles CR, Nunes MF, Queiroz MG. 
Motivation towards career choice of Correspondência para:

Brazilian freshman students in a fifteen-year Maria do Carmo Matias Freire period. J Dent Educ. 2011;75(1):115-21. e-mail: mcmfreire@ yahoo.com.br

28. Spallek H, O’Donnell JA, Yoo YIJ. Faculdade de Odontologia da Universidade Preparing faculty members for significant Federal de Goiás curricular revisions in a school of dental Av. Universitária, Praça Universitária medicine. J Dent Educ. 2010;74(3):275-88. 74605-220 Setor Universitário, Goiânia/GO

29. Freitas SFT, Calvo MCM, Lacerda JT. Saúde coletiva e novas diretrizes curriculares em odontologia: uma proposta para graduação. Trab Educ Saúde. 2012; 10(2):223-34. 